\section{SHED: A FORTRAN IV program for the analysis of small group sociometric structure}

\author{
ELLIOT NOMA \\ University of Michigan, Ann Arbor, Michigan 48109
}

and

\section{RANDALL SMITH \\ Rutgers College, New Brunswick, New Jersey 08903}

Measuring the amount and type of structure in a small group or social network is of practical and theoretical interest (Holland \& Leinhardt, 1976). Besides verifying the existence of hierarchies and cliques within the overall structure, sociometric measures often attempt to specify which models best describe the actual structure given the nature of the group, a description of group members, group size, and so on. Most measurement analyzes the pattern of positive, asymmetric relationships between pairs of individuals in a population. These data are usually reported in a sociogram, that is, a sociomatrix of $1 \mathrm{~s}$ and $0 \mathrm{~s}$. A 1 in the ijth cell means that $i$ likes $j$, $i$ gives products to $j$, or $i$ communicates with $j$. A 0 represents the absence of such a relationship.

Method of analysis is determined by the group structure model. In some situations, cliques are the structures extracted. Here, individuals of a clique have a 1 relationship to all others in the same clique (see Harary, Norman, \& Cartwright, 1965). Other times, clusters (for an overview, see Lankford, 1974) or blocks (Breiger, Boorman, \& Arabie, 1975), which are characterized as subgroups with primarily 1 relationships between individuals are the models that best reflect the group structure. Ranking the individuals (Katz, 1953) or identifying key people that bridge gaps between groups (Ross \& Harary, 1955) are other structural models.

Another measurement method is to examine all subsets of the population with a given number of individuals. This is the domain of structural balance models (Holland \& Leinhardt, 1971). Here the degree of structure is determined by examining the standard building block of the structural model, the triad: a subset of three people from the population, with a measure of the six asymmetric relationships between all pairs within the subset of three. Triad structures are the building blocks of the previously mentioned macrostructures. If the building blocks are not available, then application of a model requiring such a foundation is unjustifiable (Davis \& Leinhardt, 1971). SHED analyzes the $\mathrm{N}$ by $\mathrm{N}$ sociomatrix by sorting and counting the dyad and triad types.

Sometimes positive sentiment choices are insufficient to characterize group structures, as in the cases of "scapegoating" or "outcasts." SHED is able to analyze trichotomized sociometric data (e.g., positive, negative, and indifferent) and computes statistics using formulas developed by Hallinan (1974) and Holland and Leinhardt (1970). The program, a trichotomous analogue to Leinhardt's SOCPAC I program (Leinhardt, 1971) counts and sorts the $N(N-1)(N-2) / 6$ triads in an $\mathrm{N}$ by $\mathrm{N}$ sociomatrix into 138 nonisomorphic triad types. Statistics include observed, expected, observed minus expected, variance of expected value, and a $z$ score and significance level for each of the 138 triad types. In addition, summary statistics $\tau$ and $\tau$-split are computed for groups of critical triads (see Hallinan, 1974, and Holland \& Leinhardt, 1970, for an explanation of the $\tau$ measures). Currently, statistics may be computed for up to four linear combinations of triad frequencies during any one run.

To facilitate this analysis, we have introduced a new coding scheme for triad types. Starting with a threelevel coding of interpersonal sentiment $(2=$ positive, $1=$ indifferent or ambivalent, $0=$ negative), there are nine different types of relationships between two people if we consider both the content and orientation of the dyad (Figure 1). There are 729 triad types, of which 138 are dissimilar up to rotations and reflections. These triads are identified by a three-digit number, one digit for each dyad. The digits are assigned by starting at one individual, $\mathrm{A}$, in the triad, and recording the dyad type to Individual $B$ and the dyad type to Individual C. These are the first two digits in the triad number. The third digit is the dyad type from B to $C$. This three-digit number, however, is not unique, since permutations of

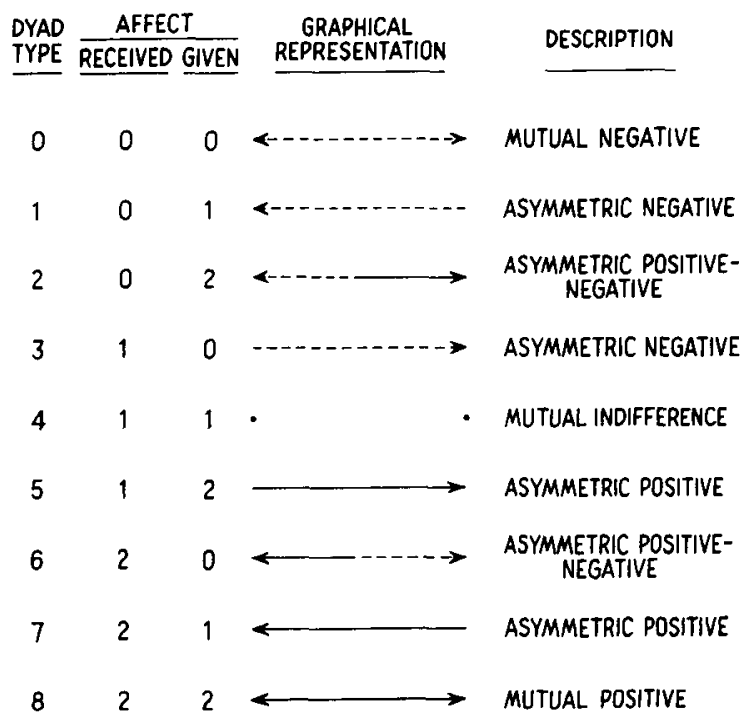

Figure 1. Nine dyad types that characterize interactions between two individuals with three levels of affect: positive, negative, and indifferent. 

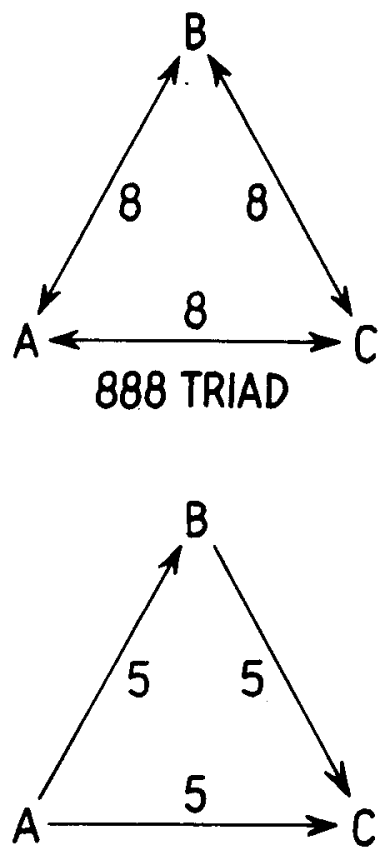

555 TRIAD

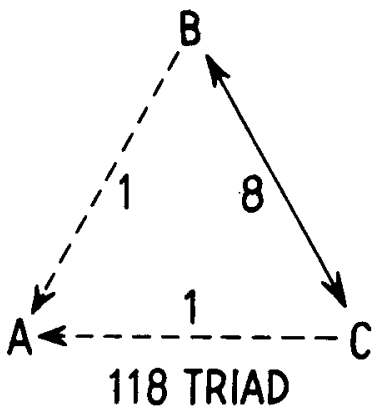

Figure 2. Three of the 138 nonisomorphic triad types. The 888 triad is required by all cliques with more than two members, and the 555 triad is required in a strict hierarchy. The 118 triad is required for "scapegoating."

the labels $\mathrm{A}, \mathrm{B}$, and $\mathrm{C}$ may result in different numbers. If different label permutations produce different codes, then the lowest number identifies a triad. Note, for instance, that the 555 triad in Figure 2 could also be called a $577,755,775,777$, or 557 triad, depending on the labeling of the nodes (persons) (i.e., the rotation and reflection of the triad). Since 555 is the smallest of the six numbers, it identifies the triad. Note also that this labeling scheme generalizes immediately to larger subsets of group structure such as tetrads and pentads. ${ }^{1}$

Options. Before running the program, the number of groups and the size of each may be specified. The program will analyze any number of groups containing $\mathrm{N} \leqslant 40$ members. Input and output options allow transformations of the data and output in card images for subsequent analysis.

Input options allow analysis using either the 16 triad types arising from a positive-nonpositive dichotomy of affect (Leinhardt, 1971), or the 138 triad types resulting from a positive-negative-indifferent trichotomy. In addition, analyses using "best friend"-other (see Hallinan \& Felmlee, 1975) or "best friend"-indifferent-negative may be requested. In all cases, weights may be supplied for each triad type to test alternative models.

Output options specify the analyses that are output and the form in which they are output. In all cases, any analysis may be output independently of other analyses either in tabular form or in card images. The card images may then be used as input to a general data analysis package (e.g., SPSS; Nie, Hull, Jenkins, Steinbrenner, \& Bent, 1975) for further processing.

Dimensions. Matrices in the program are presently dimensioned to handle 40 by 40 (or smaller) sociomatrices, but these dimensions can be altered to process larger data sets. The program requires approximately $10 \mathrm{~K}$ of core on an IBM 370/168.

Running Time. The time needed to analyze a sociomatrix is highly dependent on the number of triad types (16 or 138), and, to a lesser extent, on the size of the sociomatrix $(N \leqslant 40)$. For example, using a $\mathrm{DEC} / 10$ computer system, an analysis of a dichotomous sociomatrix (16 triad types) requires approximately $2 \mathrm{sec}$ CPU time. An analysis on a similar trichotomous sociomatrix (138 triad types) requires approximately $20 \mathrm{sec}$ CPU time.

Computer. FORTRAN IV versions of the program have been executed on an IBM 370/168 and an Amdahl $470 \mathrm{~V} / 6$ at the University of Michigan, a DEC/10 at Johns Hopkins University, and a Honeywell G635 at Dartmouth College. A BASIC version runs on a Wang 2200 , but only processes the 16 dichotomous affect triads.

Availability. Program listing and FORTRAN deck may be obtained at no cost from Elliot Noma, Psychology Department, University of Michigan, Ann Arbor, Michigan 48109, or D. Randall Smith, Rutgers College, Department of Sociology, New Brunswick, New Jersey 08903.

\section{REFERENCES}

Breiger, R. L., Boorman, S. A., \& Arabie, P. An algorithm for clustering relational data, with applications to social network analysis and comparison with multidimensional scaling. Journal of Mathematical Psychology, 1975, 12, 328-383.

Davis, J. A., \& Leinhard, S. The structure of positive interpersonal relations in small groups. In J. Berger (Ed.), Sociological theories in progress. Boston: Houghton Mifflin, 1971.

Hallinan, M. The structure of positive sentiment. Amsterdam, The Netherlands: Elsevier, 1974.

Hallinan, M., \& Felmlee, D. An analysis of intransitivity in sociometric data. Sociometry, 1975, 38, 195-212.

Harary, F., Norman, R. Z., \& Cartwright, D. Structural models: An introduction to the theory of directed graphs. New York: John Wiley, 1965. 
Holland, P. W., \& Leinhardt, S. A method for detecting structure in sociometric data. American Journal of Sociology. $1970,75,492-513$.

Holland, P. W., \& LeinhardT, S. Transitivity in structural models of small groups. Comparative Group Studies, 1971, 2. 107-124.

Holland, P. W., \& Leinhardt, S. Local structure in social networks. In D. R. Heise (Ed.), Sociological methodology, 1976. San Francisco: Jossey-Bass, 1976.

KaTz, L. A new status index derived from sociometric analysis. Psychometrika, 1953, 18, 39-43.

LANKFORD, P. M. Comparative analysis of clique identification methods. Sociometry, 1974, 37, 287-305.

LEINHARDT, S. SOCPAC I: A FORTRAN IV program for structural analysis of sociometric data. Behavioral Science, $1971,16,515-516$.

Nie, N. H., Huld, C. H., Jenkins, J. G., Steinbrenner, K., \& BENT, D. H. Statistical package for the social sciences (2nd Ed.). New York: McGraw-Hill, 1975.
Ross, I. C., \& HARARY, F. Identification of the liaison persons of an organization using the structure matrix. Management Science, 1955, 1, 251-258.

\section{NOTE}

1. This notation was adopted in preference to that introduced by Davis and Leinhardt (1971), since trichotomized (positive-indifferent-negative) relations do not lend themselves to identification by dyad type counts followed by a suffix indicating orientation.

(Received for publication July 29, 1977; revision accepted September 13,1977.) 